\title{
Determination of isotretinoin in pharmaceutical formulations by reversed-phase HPLC
}

\author{
Carla Aiolfi Guimarães ${ }^{1 *}$, Farid Menaa ${ }^{2}$, Bouzid Menaa ${ }^{3 *}$, Ivo Lebrun ${ }^{4}$, Joyce S. Quenca-Guillen ${ }^{1}$, \\ Aline Vivian Vatti Auada ${ }^{4}$, Lucildes P. Mercuri ${ }^{5}$, Paula Ferreira ${ }^{5}$, Maria Inês Rocha Miritello Santoro ${ }^{1}$ \\ ${ }^{1}$ Department of Pharmacy, Faculty of Pharmaceutical Sciences, University of São Paulo, São Paulo, Brazil; \\ ${ }^{2}$ Department of Dermatology, School of Medicine, University of Wuerzburg, Wuerzburg, Germany; \\ ${ }^{3}$ Fluorotronics, Inc., 1425 Russ. Bvld, San Diego Technology Incubator, San Diego, USA; \\ ${ }^{4}$ Department of Biochemistry and Biophysics, Butantan Institute, São Paulo, Brazil; \\ ${ }^{5}$ Department of Exact and Earth Sciences, Diadema, Federal University of São Paulo, São Paulo, Brazil. \\ Email: carlaaiolfi@usp.br; bouzid.menaa@gmail.com
}

Received 4 February 2010; revised 1 March 2010; accepted 2 March 2010.

\section{ABSTRACT}

The development of facile and rapid quantification of biologically active biomolecules such as isotretitoin in therapeutic drugs contained in many generic formulations is necessary for determining their efficiency and their quality to improve the human health care. Isotretritoin finds its applications in the maintenance of epithelial tissues. Different processes to date such as normal phase HPLC, or gas chromatrography among others are able to separate and quantify isotetroin. However, the extraction is quite complex and in the case of HPLC, the analysis requires long retention times. In such context, an isocratic reversedphase high-performance liquid chromatography (HPLC) technique coupled with an UV-vis detector is described here for easy separation and quantification of 13-cis-retinoic (isotretinoin) from soft gelatin capsule formulations. The isotretinoin was extracted from three different commercial drug samples with tetrahydrofuran (THF) solvent by a procedure that can be completed in less than 10 minutes. Subsequent separation and quantification were accomplished in less than 5 minutes under isocratic reversed-phase conditions on a Lichrospher RP18 column and a mobile phase consisting of $0.01 \%$ TFA/acetonitrile $(15 / 85, \mathrm{v} / \mathrm{v})$ at a flow rate of $1.0 \mathrm{~mL} / \mathrm{min}$. Isotretoin was detected for the three samples via its UV-vis absorbance at $342 \mathrm{~nm}$. The method was validated and the results showed good linearity, precision and accuracy for sensitive and selective quantitative determination of isotretinoin in the different pharmaceutical formulations. We found that the average isotretinoin content in two of the three commercial products fell outside the $90-110 \%$ United States Pharmacopeia specifications. Consequently, the facile extraction and the precise method for the biomole- cule quantification open up tremendous possibilities in improving the quality control of drugs which can exist as different generic brands.

Keywords: 13-Cis Retinoic Acid; Reversed-Phase Chromatography; Isotretitoin Extraction; Isotretinoin Quantification; Pharmaceuticals Formulation

\section{INTRODUCTION}

Retinoic acid is a very potent biomolecule in promoting growth and controlling differentiation and maintenance of epithelial tissue for vitamin A deficient animals. Indeed, all-trans-retinoic acid (tretinoin) appears to be active form of vitamin $\mathrm{A}$ in all tissues except the retina, and it is 10-100-fold more potent than retinol in various systems in vitro. Isomerization of this compound in the body yields to 13-cis-retinoic acid (isotretinoin), which is nearly as potent as tretinoin in many of its actions on epithelial tissues. Vitamin A and other retinoids have found wide applications in the treatment of skin disorders and may find important roles in cancer chemoprevention and therapy [1].

One of the most clinically useful vitamin A derivatives is 13-cis-retinoic acid (isotretinoin). It was approved in 1982 to treat severe recalcitrant nodular acne and remains the drug of choice for this therapeutic treatment. Isotretinoin is the only treatment that affects all the major factors involved in the pathogenesis of acne. Its exhibits several activities, in particular a capacity to decrease sebaceous gland activity, to correct the keratinisation defect in acne and to reduce the population of the bacterium Propionibacterium acnes [2].

Isotretinoin is a strong lipophilic molecule, almost insoluble in water and only partially soluble in oil. It is labile upon heating, light and air. In such conditions, these physicochemical properties impose considerable 
limitations upon formulation options. For instance, the degradation of isotretinoin (in its solid state) in the presence of air is an autocatalytic process that proceeds rapidly and a simple dry powder formulation would not have a sufficient shelf-life. A suspension in oil is more stable [2]. Suspension in oil also minimizes chemical degradation, as lipid preparations, especially those with a low peroxide number, have been shown to enhance stability [3]. Owing to the photolability and sensitivity to heat and oxidation of the retinoids, their quantitative determination in pharmaceutical is particularly important for the quality control of finished products and for stability-indicating assays [1].

Indeed, for all pharmaceutical agents, one of the most important therapeutic determinants is the amount of drug the patient is exposed to. Previous studies comparing the quality of a variety of generic with innovator products have revealed important deficiencies in the amount of active ingredient among generics [4,5]. Isotretinoin and its trans-isomer, tretinoine, are known to be teratogens that can cause fetal malformations during pregnancy for intance [6,7]. It is possible for a patient to be treated with a different isotretinoin-based generic products during a course of therapy because of an assumption that 'generic isotretinoins' are essentially identical and bioequivalent to the innovator product. Under these circumstances, the pharmaceutical quality and consistency among products become of paramount importance [2].

A number of previous methods for determination of isotretinoin have been described in the literature. The analytical method generally employed in the United State Pharmacopoeia (USP) is a normal phase HPLC system [8]. Isotretinoin has also been determined by gas chromatography in soft and hard capsules formulations [9]. A reversed phase HPLC method with fluorescence detection has been described [1] for the determination of retinoids in pharmaceutical dosage forms. Simultaneous determination of tretinoin and isotretinoin in dermatological formulations has been also reported via normal phase HPLC [10]. HPLC [1], Microcalorimetry [11], UV radiation monitor [12], thin layer chromatography (TLC) and preparative layer chromatography (PLC) [13], capillary zone electrophoresis (CZE) and micellar electrokinetic capillary chromatography (ME-KC) [14] have been also employed to study retinoid degradation products in commercial preparation and raw materials.

Although the above methods are able to separate and quantify isotretinoin, the extraction procedure is quite complex and HPLC analysis involves long retention times [10]. In that context, the aim of this study was to develop a rapid method using an isocratic high performance liquid chromatography (HPLC) coupled to UV-vis detector to determine and quantify the isotretinoin in soft capsule formulations from three commercial samples. The validation method with its precision and accuracy was checked and the isotretinoin quantification was car- ried out for three commercial products for which the content of the biomolecule was compared to the United States Pharmacopeia specifications.

\section{MATERIALS AND METHODS}

\subsection{Chemicals and Reagents}

The content of isotretinoin was determined from the concentrations of standard solutions. The standards for 13-cis-retinoic acid were from United States Pharmcopeia reference standard (USP:RS). The three commercial products were purchased from two different pharmaceutical companies (their names have been omitted for commercial reasons) named as Branded Original Product A (Product A), Generic Product B (Product B) and Generic Product $\mathrm{C}$ (Product $\mathrm{C}$ ). All three commercial soft-gel capsules contained $20 \mathrm{mg}$ of isotretinoin. Acetonitrile, trifluoroacetic acid (TFA), tetrahydrofuran (THF) were HPLC grade and purchased from Merck (USA).

\subsection{Laboratory Precaution}

All experiments were performed using amber glass volumetric flasks under yellow light conditions to avoid degradation of isotretinoin.

\subsection{HPLC Instrumentation and Conditions}

The HPLC system consisting of Agilent model 1100 (Agilent, USA) connected to a UV/Visible absorbance detector (agilent) was used for all separation. Chromatographic separations were performed using reversedphase chromatographic using a HPLC column [Lichrospher RP18 $(5 \mu \mathrm{m}, 125 \times 4 \mathrm{~mm}$ i.d., Merck, USA $)]$. Isotretinoin was detected by the UV-vis absorbance at $342 \mathrm{~nm}[8]$ with the sensitivity set at 0.1 aborbance unit full scale (Aufs).

The mobile phase used was composed of $0.01 \%$ trifluoroacetic acid and acetonitrile $(15 / 85, \mathrm{v} / \mathrm{v})$ at a flow rate of $1.0 \mathrm{~mL} / \mathrm{min}$. The injection volume was $20 \mu \mathrm{L}$ and the column was maintained at $40^{\circ} \mathrm{C}$.

\subsection{Standard Preparation Procedures}

Stock solutions were prepared by dissolving the appropriate amounts of isotretinoin in acetonitrile. A set of working standard solutions was produced by diluting aliquots of the stock solutions to give the desired concentrations of the analytes. For the method linearity assessment, the concentration range was 5.0 to $30.0 \mu \mathrm{g} / \mathrm{mL}$ (the concentrations were determined using the calibration graphs. The standard solutions were stable for at least 3 days at $4^{\circ} \mathrm{C}$ ).

\subsection{Extraction of Soft-Gel Capsules Formulations}

Isotretinoin capsules were opened carefully using a sharp blade and the content of these capsules was ex- 
tracted directly with THF solvent. The solution containing isotretinoin was shaked for $5 \mathrm{~min} .1 \mathrm{~mL}$ of aliquot for the resulting solution was diluted in acetonitrile to obtain the final solution concentration $(20 \mu \mathrm{g} / \mathrm{mL})$ that was analyzed by HPLC in comparison with the appropriate standard solution.

\subsection{Quantification of Isotretinoin}

A standard curve was constructed by injecting samples containing isotretinoin standard at concentrations ranging from 5.0 to $30.0 \mu \mathrm{g} / \mathrm{mL}$. The peak area was determined and plotted versus the concentration of isotretinoin. For the recovery studies, known volumes of isotretinoin standard solutions were analyzed, and the absolute recovery was calculated by comparing the peak area obtained from isotretinoin in the commercial capsule with the peak area of samples derived from the standard solutions.

\section{RESULTS AND DISCUSSION}

The objective of this work was to develop a rapid me- thod using an isocratic high performance liquid chromatography (HPLC) system to determine quantitatively the isotretinoin in hard gelatin capsule formulations. In order to validate an efficient method for analysis of drugs in pharmaceutical formulations, preliminary tests were performed with the objective to select adequate and optimum conditions. Parameters such as detection wavelength, ideal mobile phase and their proportions, concentration of the standard solutions, and flow rate were exhaustively tested and the quantitative determination of isotretoin in the gel capsules was made possible under the ideal conditions described in this paper. The proposed method is simple and do not involve laborious time-consuming sample preparation.

\subsection{Chromatography}

The conditions for a rapid and simple HPLC separation with UV detection were developed using an isocratic elution with a mobile phase composed of $0.01 \%$ trifluoroacetic acid and acetonitrile $(15 / 85, \mathrm{v} / \mathrm{v})$. These conditions gave well resolved sharp peaks (Figure 1) for

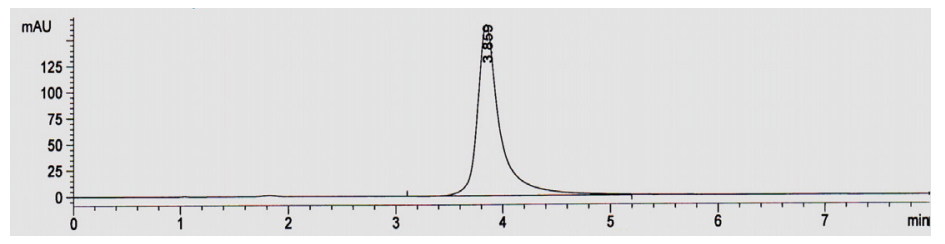

(a)



(c)

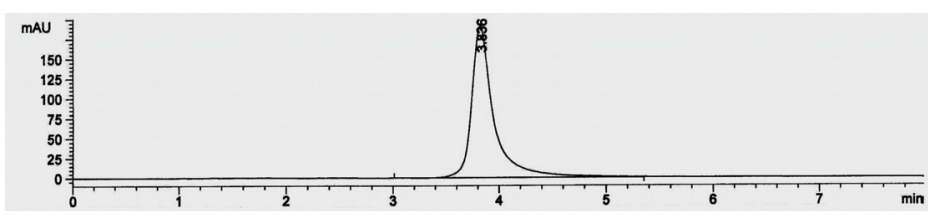

(d)

Elution time (min)

Figure 1. Chromatogram of samples. (a) isotretinoin standard $(20 \mu \mathrm{g} / \mathrm{mL})$; (b) Product A $(20 \mu \mathrm{g} / \mathrm{mL})$; (c) Product B $(20 \mu \mathrm{g} / \mathrm{mL})$; (d) Product C $(20 \mu \mathrm{g} / \mathrm{mL})$. Conditions: Lichrospher RP18 column $(5 \mu \mathrm{m}, 125 \times 4 \mathrm{~mm}$ i.d., Merck), mobile phase $0.01 \%$ TFA /acetonitrile $(15 / 85, \mathrm{v} / \mathrm{v})$, flow rate $1.0 \mathrm{~mL} / \mathrm{min}$, UV detection at $342 \mathrm{~nm}$ and ambient temperature $\left(24 \pm 2^{\circ} \mathrm{C}\right)$. 
the isotretinoin commercial formulations but also for the isotretinoin standards. The retention time was of approximately $3.8 \mathrm{~min}$. The shorter elution time makes the method especially useful for routine analysis of isotretinoin in pharmaceutical formulations. The validation experiments as described in the following section were completed to determine if the method could achieve the reproducibility and accuracy required for analysis of formulations.

\subsection{Method Validation}

\subsubsection{Method Validation}

The quantification of the chromatogram was performed using the peak area of isotretinoin standards for known content of isotretinoin and as references for the determination of the content for the commercial samples to which we determined the peak areas of their respective chromatograms. Five standard solutions ranging from 5.0 to $30.0 \mu \mathrm{g} / \mathrm{mL}$ in concentration (three replicates each), were injected into the HPLC system. The calibration graph was obtained by plotting the peak areas of the standard solutions against the theoretical standard concentrations. The linearity was evaluated by linear leastsquares regression analysis. The correlation coefficient obtain with linear regression of curve was 0.997 . Statistical analysis indicated excellent linearity as shown in Table 1.

\subsubsection{Accuracy}

To evaluate the accuracy of the proposed method, recovery tests were carried out with all samples. The measurements were performed by adding known amounts of standard solutions to sample followed by analysis using the proposed method. The recovery values obtained were 101.2, 99.4 and $99.3 \%$ (why don't you have $100 \%$ ) for the three soft gelatin capsule formulations, confirming the accuracy of the proposed method. The percentage of recovery results are presented in Table 1.

\subsubsection{Precision}

The precision of proposed method was evaluated through intra-day repeatability of responses after replicate (n $=10)$ injection of sample solutions $(20.0 \mu \mathrm{g} / \mathrm{mL})$. The precision is expressed as the relative standard deviation (RSD) amongst responses. The standard deviation amongst replicate responses and relative standard deviation values (RSD) were less than $1.0 \%$, indicating precision of the method. The statistical data results obtained in the analysis of commercially available samples are shown in Table 2.

According to the results obtained only one formulation (Product A (branded named)), was found to be good agreement with the claimed content of the drugs. Indeed, the average percentage of isotretinoin content (Table 3) for the Product A was evaluated to $105 \pm 0.05 \%$ which fits with United States Pharmacopeia (USP) specifications. In contrast, the content for the generic products formulations Products (Product B and Product C), fell outside the United States Pharmacopeia (USP) specifications. The content percentage of isotretinoin was found to be $128.5 \pm 0.08$ and $115.5 \pm 0.04$ in Product $\mathrm{B}$ and Product C, respectively. The USP XXX [8] monograph for isotretinoin capsules states that the capsules must contain not less than $90.0 \%$ and not more than $110 \%$ of the labeled amounts of isotretinoin. The results and the accuracy of the method shows that it is necessary to control the content of the active biomolecule for drug safety, but also that the precision of the method and rapid acquisition of the data can be a plus for pharmaceutical industries for the quality control of their products.

Table 1. Statistical results of linear regression analysis in the determination of isotretinoin by proposed method.

\begin{tabular}{cc}
\hline Statistical parametrs & Results \\
\hline Intercept of curve (b) & -328.09 \\
Slope of curve (a) & 138.86 \\
Linear correlation coefficient (r) & 0.997 \\
\hline
\end{tabular}

Table 2. Recovery data of standard solutions added to the samples analyzed by using the proposed HPLC method.

\begin{tabular}{ccccc}
\hline Sample & $\begin{array}{c}\text { Amount } \\
\text { added } \\
(\boldsymbol{\mu g} / \mathbf{m L})\end{array}$ & $\begin{array}{c}\text { Amount } \\
\text { found* } \\
(\boldsymbol{\mu g} / \mathbf{m L})\end{array}$ & Recovery(\%) & $\begin{array}{c}\text { Mean } \\
\text { recov- } \\
\mathbf{e r y}(\%)\end{array}$ \\
\hline Product & 25.0 & 26.0 & 100.0 & \\
A & 30.0 & 31.3 & 103.0 & 101.2 \\
& 35.0 & 36.1 & 100.6 & \\
Product & 25.0 & 30.6 & 98.0 & \\
B & 30.0 & 35.8 & 101.0 & 99.4 \\
& 35.0 & 40.6 & 99.3 & \\
& 25.0 & 28.2 & 102.0 & \\
Product & 30.0 & 32.9 & 98.0 & 99.3 \\
C & 35.0 & 37.8 & 98.0 & \\
\hline
\end{tabular}

*Avarage of three determination

Table 3. Statistical data obtained in the analysis of samples using the proposed method.

\begin{tabular}{ccccc}
\hline Sample & $\begin{array}{c}\text { Declared theoretical } \\
\text { concentration }(\boldsymbol{\mu} / \mathbf{m L})\end{array}$ & $\begin{array}{c}\text { Found experimental } \\
\text { concentration }(\boldsymbol{\mu g} / \mathbf{m L})\end{array}$ & Content $(\%)$ & RSD (*) (\%) \\
\hline Product A & 20.0 & 21.0 & 105.0 & 0.43 \\
Product B & 20.0 & 25.7 & 128.5 & 0.70 \\
Product C & 20.0 & 23.1 & 115.5 & 0.30 \\
\hline
\end{tabular}

(*) Average of ten determination (**) $95.0 \%$ confidence level. 


\section{CONCLUSIONS}

The proposed HPLC methods for quantitative determination showed good linearity, precision and accuracy for sensitive and selective quantitative determination of isotretinoin in pharmaceutical formulations. This method is not time-consuming and is easy to perform in any laboratory. We ultimately show, according the United States Pharmacopeia specifications that the average amount of isotretinoin in 2 of the 3 commercial products ranged outside the $90-110 \%$. Safe use of isotretinoin, particularly with respect to teratogenicity, remains a central issue for therapeutics development. Therefore, it is important, that future research determines a comparative study with pharmaceutical quality of isotretinoin products on patient exposure to isotretinoin by comparative bioequivalence tests.

\section{ACKNOWLEDGEMENTS}

The authors acknowledge the CNPq (National Counsel of Technological and Scientific Development) CAPES (Foundation to Higher Level People Improvement) and FAPESP (Research Support Foundation in the State of São Paulo) for the financial support.

\section{REFERENCES}

[1] Gatti, R., Gioia, M.G. and Cavrini, V. (2000) Analysis and stability study of retinoids in pharmaceuticals by fluorescence. Journal of Pharmaceutical and Biomedical Analysis, 23(1), 147-159.

[2] Taylor, P.W. and Keenan, M.H.J. (2006) Pharmaceutical quality of generic isotretinoin products, compared with Roaccutane. Current Medical Research and Opinion, 22(3), 603-615.

[3] Ioele, G., Cione, E., Risoli, A., Genchi G. and Ragno, G. (2005) Accelerated photostability study of tretinoin and isotretinoin in liposomes formulations. International Journal of Pharmaceutics, 60(1-2), 251-260.

[4] Wetterich, U. and Mutschler, E. (1995) Quality of cefotaxine sodium preparations. Arzneimittel-Forschung Drug
Research, 45(1), 74-80.

[5] Lambert, P.A. and Conway, B.R. (2003) Pharmaceutical quality of ceftriaxone generic products compared with Rocephin. Journal Chemotherapy, 15(4), 357-368.

[6] Lammer, E.J., Chen, D.T., Hoar, R.M., Agnish, N.D., Benke, P.J. and Braun, J.T. (1985) Retinoic acid embryopathy. New England Journal of Medicine, 313(14), 837-841.

[7] Willhite, C.C., Wier, P.J. and Berry, D.L. (1989) Dose response and structure-activity considerations in retinoidinduced dysmor phogênesis. Critical Reviews in Toxicology, 20(2), 689-695.

[8] (2007) United States pharmacopeia. XXX, Pharmacopeia Convention, Maryland, 2428-2429.

[9] Lima, E.M., Diniz, D.G. and Antoniosi-Filho, N.R. (2005) Development of a gas chromatography method for the determination of isotretinoin and its degradation products in pharmaceuticals. Journal of Pharmaceutical of Biomedical Analysis, 38(4), 678-685.

[10] Tashtoush, M.B. Jacobson, E.L. and Jacobson, M.K. (2007) A rapid HPLC method for simultaneous determination of tretinoin and isotretinoin in dermatological formulations. Journal of Pharmaceutical and Biomedical Analysis, 43(3), 859-864.

[11] Tan, X., Meltzer, N. and Lindenbaum, S. (1992) Solidstate stability studies of 13-cis-retinoic acid and alltransretinoic acid using microcalorimetry and HPLC analysis. Pharmaceutical Research, 9(9), 1203-1208.

[12] Allwood, M.C. and Plane, J.H. (1984) The degradation of vitamin A exposed to ultraviolet radiation. International Journal of Pharmaceutics, 19(2), 207-213.

[13] Crank, G. and Pardijanto, M.S. (1995) Photooxidations and photosensitized oxidations of vitamin A and its palmitate ester. Journal of Photochemistry and Photobiology A: Chemistry, 85(1-2), 93-100.

[14] Bempong, D.K., Honigberg, I.L. and Meltzer, N.M. (1993) Separation of 13-cis and all-trans retinoic acid and their photodegradable products using capillary zone electrophoresis and micellar electrokinetic chromatography (MEC). Journal of Pharmaceutical and Biomedical Analysis, 11(9), 829-833. 